\title{
A multicenter study on the effect of continuous hemodiafiltration intensity on antibiotic pharmacokinetics
}

\author{
Darren M Roberts ${ }^{1 *}$, Xin Liu ${ }^{2,3,4}$, Jason A Roberts ${ }^{1,5}$, Priya Nair ${ }^{6}$, Louise Cole ${ }^{7}$, Michael S Roberts ${ }^{2,3,4}$, Jeffrey Lipman ${ }^{1,5}$,
} Rinaldo Bellomo ${ }^{8}$ and On behalf of the RENAL Replacement Therapy Study Investigators

\begin{abstract}
Introduction: Continuous renal replacement therapy (CRRT) may alter antibiotic pharmacokinetics and increase the risk of incorrect dosing. In a nested cohort within a large randomized controlled trial, we assessed the effect of higher ( $40 \mathrm{~mL} / \mathrm{kg}$ per hour) and lower ( $25 \mathrm{~mL} / \mathrm{kg}$ per hour) intensity CRRT on antibiotic pharmacokinetics.

Methods: We collected serial blood samples to measure ciprofloxacin, meropenem, piperacillin-tazobactam, and vancomycin levels. We calculated extracorporeal clearance $(\mathrm{CL})$, systemic $\mathrm{CL}$, and volume of distribution (Vd) by non-linear mixed-effects modelling. We assessed the influence of CRRT intensity and other patient factors on antibiotic pharmacokinetics.
\end{abstract}

Results: We studied 24 patients who provided 179 pairs of samples. Extracorporeal CL increased with higher-intensity CRRT but the increase was significant for vancomycin only (mean 28 versus $22 \mathrm{~mL} /$ minute; $P=0.0003$ ). At any given prescribed CRRT effluent rate, extracorporeal CL of individual antibiotics varied widely, and the effluent-to-plasma concentration ratio decreased with increasing effluent flow. Overall, systemic CL varied to a greater extent than Vd, particularly for meropenem, piperacillin, and tazobactam, and large intra-individual differences were also observed. CRRT dose did not influence overall (systemic) CL, Vd, or half-life. The proportion of systemic CL due to CRRT varied widely and was high in some cases.

Conclusions: In patients receiving CRRT, there is great variability in antibiotic pharmacokinetics, which complicates an empiric approach to dosing and suggests the need for therapeutic drug monitoring. More research is required to investigate the apparent relative decrease in clearance at higher CRRT effluent rates.

Trial registration: ClinicalTrials.gov NCT00221013. Registered 14 September 2005.

\section{Introduction}

Bacterial sepsis is common and increases mortality in critically ill patients with acute kidney injury (AKI) [1]. The administration of antibiotics is a key component of therapy in these patients [2]. The influence of antibiotic concentration on bacterial kill has been determined in vitro and is the basis of current approaches to antibiotic dosing [3]. Logically, incorrect dosing secondary to a poor understanding of pharmacokinetics may contribute to adverse

\footnotetext{
* Correspondence: darren.roberts@uq.edu.au

'Burns Trauma and Critical Care Research Centre, The University of Queensland, Level 3 Ned Hanlon Building, Royal Brisbane and Women's Hospital, Butterfield Street, Brisbane, Queensland 4029, Australia Full list of author information is available at the end of the article
}

outcomes, including treatment failure [4] or the escalation of antibiotic resistance [5].

Key aspects of pharmacokinetics like antibiotic clearance $(\mathrm{CL})$ and volume of distribution $(\mathrm{Vd})$ are altered in critically ill patients with AKI because of loss or renal clearance, volume expansion, and interventions such as vasopressors and continuous renal replacement therapy (CRRT) [6,7]. Moreover, extracorporeal CL during CRRT is influenced by the physicochemical and pharmacokinetic properties of the antibiotic, blood flow, dialysate flow, and ultrafiltration rate and by membrane fouling and filter clotting [8]. These variables may lead to sub-therapeutic blood concentrations and contribute to treatment failure [9-11]. However, data on antibiotic pharmacokinetics 
during CRRT are limited in scope and detail [12], making correct prescription problematic. Accordingly, in patients treated with CRRT of different intensities, we aimed to evaluate variability in $\mathrm{CL}$ and $\mathrm{Vd}$ and to assess the effect of the CRRT prescription on extracorporeal and systemic antibiotic CL and Vd.

\section{Methods}

\section{Clinical}

This was a nested cohort prospective multicenter observational pharmacokinetic study within a large randomized controlled trial of CRRT intensity (trial registration: ClinicalTrials.gov NCT00221013, registered 14. September 2005) [13]. Inclusion criteria for this study, known as the Randomized Evaluation of Normal vs. Augmented Level of CRRT (RENAL) study, have been previously published and are outlined in the online supplement (Additional file 1).

In brief, patients were randomly assigned to receive post-dilutional hemodiafiltration as either a higher (40 $\mathrm{mL} / \mathrm{kg}$ body weight/hour effluent flow rate) or lower $(25 \mathrm{~mL} / \mathrm{kg}$ body weight/hour effluent flow rate) intensity rate using equal size filters with polyacrylonitrile membranes. By protocol, the target prescribed effluent flow was achieved through an equal contribution of dialysate flow and ultrafiltration. Blood flow was $200 \mathrm{~mL} /$ minute for all study patients except two (150 or $180 \mathrm{~mL} /$ minute).

Four hospitals in three separate geographical regions participated in this study. Institutional review boards approved the study, and informed consent and demographic, clinical, and laboratory data were obtained [13]; see the online supplement (Additional file 1) for more details.

Pharmacokinetic sampling occurred at three time points each day: (1) immediately before antibiotic dosing, (2) after completion of their intravenous infusion, and (3) at 4 hours after completion of infusion. A patient could participate on more than one occasion but on different days. The time of sampling was recorded exactly, and at each time point, a pre-filter blood sample and CRRT effluent sample were obtained simultaneously. Plasma was separated by centrifugation, immediately frozen, and stored at $-70^{\circ} \mathrm{C}$ until analysis.

\section{Laboratory techniques}

The plasma and effluent concentrations of ciprofloxacin (molecular weight (MW) 331.3), meropenem (MW 383.5), piperacillin (MW 517.6), and tazobactam (MW 300.3) were determined by using a validated liquid chromatography with tandem mass spectrometry method that has been described previously [9]; see online supplement (Additional file 1) for more details. The plasma concentrations of vancomycin (MW 1449.3), urea (MW 60.1), and creatinine (MW 113.1) were determined by using commercial assays by the Chemical Pathology Laboratory, Princess Alexandra Hospital, Queensland Health Scientific and Forensic Services, Brisbane, Australia.

\section{Pharmacokinetic calculations}

The antibiotic $\mathrm{CL}$ from hemodiafiltration $\left(\mathrm{CL}_{\mathrm{HDF}}\right)$ was calculated at each time point on the basis of drug recovery in the CRRT effluent by using a standard formula $[8,14]$ :

$$
\mathrm{CL}_{\mathrm{HDF}}=\left(\mathrm{C}_{\mathrm{E}} / \mathrm{C}_{\mathrm{P}}\right) \times \mathrm{Q}_{\mathrm{E}}
$$

where $C_{E}$ is the solute concentration in CRRT effluent, $Q_{E}$ the prescribed CRRT effluent flow, and $C_{P}$ the solute concentration in plasma. The $C_{E} / C_{P}$ ratio is referred to as effluent or dialysate saturation [14] or the saturation coefficient (Sd) [8]. The Sd is the diafiltration equivalent of sieving coefficient, which is calculated in the same way during hemofiltration. The Sd was also calculated for urea and creatinine as a measure of filter membrane function.

Using pre-filter plasma concentrations at the three time points, the systemic $\mathrm{CL}\left(\mathrm{CL}_{\mathrm{s}}\right)$ and $\mathrm{Vd}$ were calculated. These time points were chosen so that the first and second points related to $\mathrm{Vd}$ and the second and third related to $\mathrm{CL}_{\mathrm{s}}$. The antibiotic plasma concentration-time data were fitted to one-, two-, or three-compartment models by non-linear mixed-effects population modelling (NONMEM software version 6.1; GloboMax LLC, Hanover, MD, USA) [15]. A Digital Fortran compiler was used, and runs were executed by using Wings for NONMEM (WFN) version 616.

Data were analyzed by using the first-order conditional estimation method with interaction. Between-subject variability was calculated by using an exponential variability model. Residual unexplained variability was tested by using exponential or additive random error or both. Visual inspection of diagnostic scatter plots and the NONMEM objective function value (OFV) were used to evaluate goodness of fit. Statistical comparison of nested models was undertaken in the NONMEM program on the basis of a chi-square test of the difference in OFV. A decrease in the OFV of 3.84 units $(P<0.05)$ was considered statistically significant.

The influence of the properties of a given antibiotic was assessed by using meropenem and vancomycin because they were the most commonly used antibiotics in this series and differed in terms of size (MW 438 versus 1,486 Daltons, respectively) and protein binding (less than $10 \%$ versus $30 \%$, respectively [6]).

The systemic elimination half-life $\left(\mathrm{T}_{1 / 2}\right)$ was calculated as follows:

$$
\mathrm{T}_{1 / 2}=(0.693 \times \mathrm{Vd}) / \mathrm{CL}_{\mathrm{s}}
$$

The proportion of $\mathrm{CL}_{\mathrm{s}}$ attributed to hemodiafiltration was calculated by: 


$$
\% \mathrm{CL}=\left(\mathrm{CL}_{\mathrm{HDF}} / \mathrm{CL}_{\mathrm{s}}\right) \times 100 .
$$

In the case of $\mathrm{CL}_{\mathrm{HDF}}$ being determined on multiple occasions in the same patient during the time period when $\mathrm{CL}_{\mathrm{s}}$ was calculated, \%CL was determined by using the mean $\mathrm{CL}_{\mathrm{HDF}}$.

\section{Statistical calculations}

Statistical analyses were conducted according to the normality of data which was determined by using the D'Agostino and Pearson omnibus normality test. Nonparametric data were subject to the Mann-Whitney and Spearman $\mathrm{r}$ correlation tests, whereas parametric data were compared by using the Student $t$ test (with Welch's correction when variances were not equal) and Pearson $r$ correlation test. The goodness of fit $\left(r^{2}\right)$ of statistically significant correlations was determined by using linear regression. All regressions and statistics were conducted by using GraphPad Prism version 5.03 for Windows (GraphPad Software, San Diego, CA, USA), and a $P$ value of less than 0.05 was considered statistically significant.

\section{Results}

Sampling occurred at 179 time points in 24 patients: ciprofloxacin (19), meropenem (65), piperacillin-tazobactam (29), and vancomycin (66). The demographics, baseline clinical characteristics, and other treatments for the 24 study patients have been presented previously [9]. Briefly, mean age \pm standard deviation was $62 \pm 17$ years, most were men (16:8), and mean weight was $82 \pm 17 \mathrm{~kg}$. Thirteen patients were diagnosed with sepsis, and edema was present in 12 patients. Admission APACHE (Acute Physiology and Chronic Health Evaluation) III and SOFA (Sequential Organ Failure Assessment) scores were $103 \pm$ 17 and $11.0 \pm 3.0$, respectively. Ventilatory support was required in $75 \%$ of patients, and 90 -day mortality was $42 \%$. The serum creatinine concentration at randomization was $273 \pm 123 \mu \mathrm{mol} / \mathrm{L}$, and oligo-anuria was present in eight patients. The prescribed effluent flow rates in the higher- and lower-intensity groups were a median (interquartile range, IQR) of 2,800 (2,600 to 3,100) $\mathrm{mL} /$ hour and 2,130 (2,000 to 2,375$) \mathrm{mL} /$ hour, respectively $(P<0.0001)$.

\section{Extracorporeal antibiotic removal}

The antibiotic pre-filter plasma concentration correlated with the CRRT effluent concentration for each of the antibiotics (online supplement Figure S1; see Additional file 1), and Sd was less than 1.0 in $94 \%$ of samples. The influence of CRRT intensity on antibiotic $\mathrm{CL}_{\mathrm{HDF}}$ is shown in Table 1. In general, $C L_{H D F}$ increased with higherintensity CRRT, but this effect was significant for vancomycin only (mean 28 versus $22 \mathrm{~mL} /$ minute; $P=0.0003$, Student $t$ test). Wide standard deviations or IQRs were noted in each case (Table 1). A correlation was noted between the prescribed effluent flow rate and $\mathrm{CL}_{\mathrm{HDF}}$ for vancomycin but not for other antibiotics (online supplement Figure S2; see Additional file 1).

The mean $\mathrm{CL}_{\mathrm{HDF}}$ of meropenem and vancomycin did not differ significantly: higher intensity $23 \pm 13$ versus $28 \pm 7 \mathrm{~mL} /$ minute $(P=0.0610)$ and lower intensity $22 \pm 5$ versus $21 \pm 9 \mathrm{~mL} /$ minute $(P=0.6899)$, respectively. The $\mathrm{CL}_{\mathrm{HDF}}$ of piperacillin was less than that of tazobactam, such that the median $\mathrm{CL}_{\mathrm{HDF}}$ ratio of piperacillin to tazobactam was 0.56 (IQR 0.39 to 0.72 ). The median ratio of the plasma concentrations of piperacillin to tazobactam was 14.9 (IQR 10.7 to 23.2, range 7.5 to 41.7 ). The Sd of vancomycin and meropenem was not consistently influenced by the albumin concentration or hematocrit (online supplement Figure S3; see Additional file 1).

\section{Extracorporeal uremic solute removal}

Urea $\mathrm{CL}_{\mathrm{HDF}}$ was significantly higher in the higherintensity group (mean $41 \pm 14$ versus $33 \pm 7 \mathrm{~mL} /$ minute, $P=0.0002$ ); the same was observed for creatinine $C_{\mathrm{HDF}}$ (mean $40 \pm 13$ versus $31 \pm 7 \mathrm{~mL} /$ minute, $P=0.0003$ ). A correlation was noted between the prescribed effluent flow rate and $\mathrm{CL}_{\mathrm{HDF}}$ for urea and creatinine (online supplement Figure S4; see Additional file 1). However, despite the lack of correlation between effluent flow rate and creatinine $\mathrm{Sd}$, a negative correlation was noted with urea $\mathrm{Sd}$ and effluent flow rate (Figure 1). Moreover, the urea and creatinine Sd varied markedly within comparable effluent flow rates. A significant association between the Sd of urea, creatinine, meropenem, or vancomycin with the age of CRRT filter was not observed (online supplement Figure S5; see Additional file 1). A positive and significant correlation was noted with the Sd of both urea and creatinine for vancomycin (Figure 2) but not meropenem (online supplement Figure S6; see Additional file 1).

\section{Systemic clearance and volume of distribution}

The results of the population pharmacokinetic model development and evaluation are outlined in the online supplement (Additional file 1). The estimates of $\mathrm{CL}_{\mathrm{s}}$ and $\mathrm{Vd}$ show that inter-individual differences were significant for $\mathrm{CL}_{\mathrm{s}}-$ coefficient of variation $(\mathrm{CV} \%) 10.0$ for ciprofloxacin and 52.3 for tazobactam-and Vd (CV\% 24.9 to 37.8 ) (Table 2).

The net effect of this variability is shown by using simulation in the case of meropenem, in which an approximate dose-response relationship is observed, but despite a twofold difference in dose, the concentrationtime curves are not clearly separated (Figure 3 ).

In the case of vancomycin and meropenem, the prescribed CRRT effluent rate did not significantly influence $\mathrm{CL}_{\mathrm{s}}$ (online supplement Figure S7; Additional file 1), Vd, or elimination half-life (data not shown). 
Table 1 The effect of continuous renal replacement therapy intensity on extracorporeal antibiotic clearance

\begin{tabular}{|c|c|c|c|c|}
\hline Antibiotic & & Antibiotic clearance, higher intensity & Antibiotic clearance, lower intensity & $P$ value \\
\hline \multirow[t]{2}{*}{ Ciprofloxacin } & Median (IQR) & $19(13-24) ; n=12$ & $17(16-20) ; n=7$ & 0.5139 \\
\hline & Mean (SD) & $19 \pm 8$ & $17 \pm 3$ & \\
\hline \multirow[t]{2}{*}{ Meropenem } & Median (IQR) & $23(16-29)$ & $21(15-28)$ & $0.4802^{\mathrm{a}}$ \\
\hline & Mean (SD) & $23 \pm 13 ; n=35$ & $21 \pm 9 ; n=28$ & \\
\hline \multirow[t]{2}{*}{ Piperacillin } & Median (IQR) & $22(21-31)$ & $24(17-31)$ & $0.9091^{\mathrm{a}}$ \\
\hline & Mean (SD) & $25 \pm 10 ; n=11$ & $26 \pm 12 ; n=17$ & \\
\hline \multirow[t]{2}{*}{ Tazobactam } & Median (IQR) & $37(34-49)$ & $56(41-66)$ & $0.0642^{a, b}$ \\
\hline & Mean (SD) & $38 \pm 13 ; n=11$ & $53 \pm 24 ; n=17$ & \\
\hline \multirow[t]{2}{*}{ Vancomycin } & Median (IQR) & $28(24-33)$ & $21(19-25)$ & $<0.0001^{a, b}$ \\
\hline & Mean (SD) & $28 \pm 7 ; n=35$ & $22 \pm 5 ; n=31$ & \\
\hline
\end{tabular}

${ }^{a}$ Statistical significance was determined by using the $t$ test because the data were considered normally distributed; the Mann-Whitney test was used for the remaining analyses. ${ }^{\mathrm{b}} \mathrm{P}<0.05$. IQR, interquartile range; $\mathrm{SD}$, standard deviation.

\section{Relationship between systemic and extracorporeal clearance}

The contribution of $\mathrm{CL}_{\mathrm{HDF}}$ to the calculated $\mathrm{CL}_{\mathrm{s}}$ also varied widely. The medians and IQRs were ciprofloxacin $32 \%$ ( $29 \%$ to $34 \%$ ), meropenem $66 \%$ ( $42 \%$ to $76 \%$ ), piperacillin $23 \%$ (19\% to $27 \%$ ), tazobactam $49 \%$ (39\% to $57 \%$ ), and vancomycin $92 \%$ (84\% to $109 \%$ ).

\section{Inter-occasional pharmacokinetic variability}

The concentration-time profile, clinical information, and pharmacokinetic parameters for two of the patients who provided samples on multiple days are shown in Figure 4. Much day-to-day variability in antibiotic pharmacokinetics was noted, although each patient received a fixed dosage of CRRT.

\section{Discussion}

\section{Key findings}

In this multicenter pharmacokinetic study of four antibiotics and tazobactam, the prescribed intensity of CRRT did not adequately predict $\mathrm{CL}_{\mathrm{HDF}}$ or $\mathrm{Sd}, \mathrm{CL}_{\mathrm{s}}, \mathrm{Vd}$, or halflife. This did not appear to be explained by the age of the filter, hematocrit, or serum albumin concentration. A correlation was noted between antibiotic concentration in the effluent and pre-filter plasma samples, where the effluent concentration was lower than that of plasma. Using urea and creatinine as biomarkers of membrane function, despite a correlation between effluent flow and $\mathrm{CL}_{\mathrm{HDF}}$, we detected failure of equilibration between dialysate and plasma during higher CRRT intensity. The correlation between the $\mathrm{Sd}$ of urea or creatinine with antibiotics was inconsistent, limiting their role as a surrogate measures for clearance.

\section{Principles of extracorporeal solute removal}

The influence of components of the prescription of an extracorporeal treatment was recently reviewed [38]. In the context of CRRT, effluent flow is the rate-limiting step because it is slower than blood flow, so clearance is anticipated to positively correlate with effluent flow rate. Furthermore, clearance by convection is dose-dependent and this is the most efficient method of removing larger molecules. Smaller molecules are also removed efficiently by diffusion; however, the effect of diffusion is non-linear
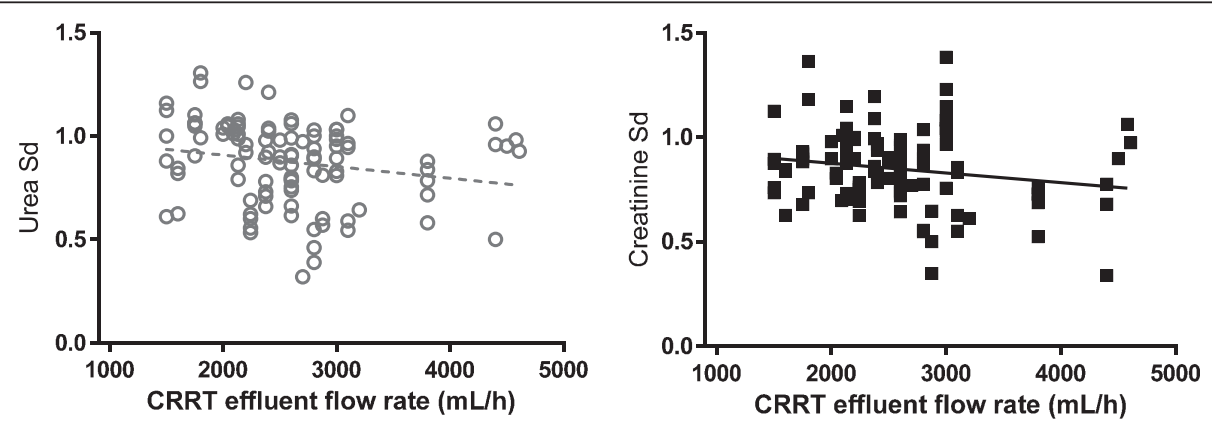

Figure 1 Marked variability but negative association between the saturation coefficients (Sd) of urea (significant) and creatinine (non-significant) and CRRT effluent flow rate. Urea: Pearson $r=-0.2016, P=0.0383, r^{2}=0.04063, n=106$ pairs; creatinine: Pearson $r=-0.1790$, $P=0.0704, \mathrm{n}=103$ pairs. CRRT, continuous renal replacement therapy. 

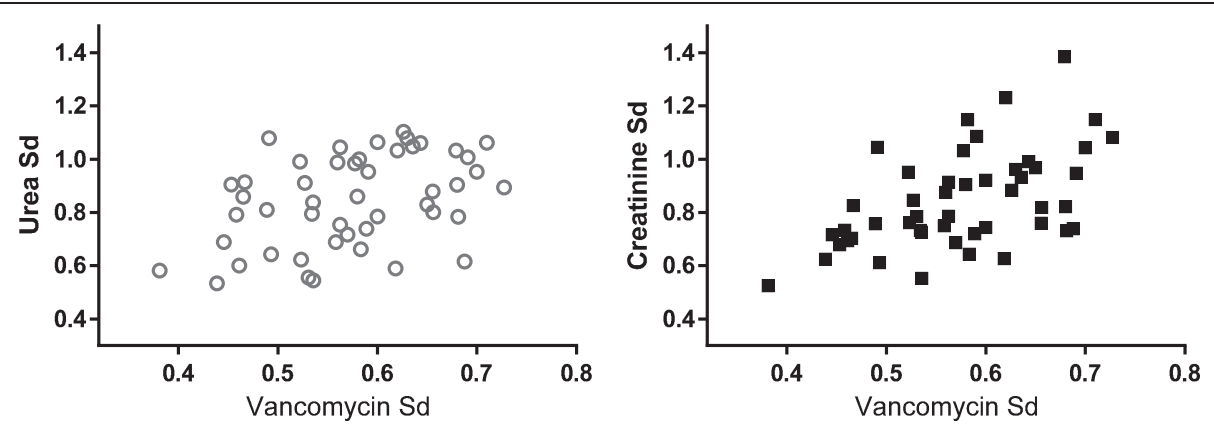

Figure 2 Positive correlation between the saturation coefficient (Sd) of vancomycin with both urea and creatinine. Urea: Spearman $r=0.3817, P=0.0074, n=48$ pairs; creatinine: Spearman $r=0.5297, P=0.0001, n=48$ pairs.

at higher effluent flow rates and this may compromise clearance. Post-filter replacement maximizes removal but this may be at the expense of the life of the filter [39].

Our results suggest that these simple and logical relationships may not be observed in clinical practice. In particular, in our study, the effect of prescribed CRRT effluent flow rate on antibiotic clearance was not consistently dose-dependent. Others have reported similar findings, including a higher Sd for beta-2 microglobulin (MW 11,000) with increasing ultrafiltration rates, and have observed that varying the proportion of dialysate and ultrafiltrate flows in hemodiafiltration influences the Sd of different solutes variably [40].

Factors that may interfere with extracorporeal solute removal A correlation was noted between $\mathrm{CL}_{\mathrm{HDF}}$ and effluent flow for vancomycin, creatinine, and urea but not for other antibiotics. These observations, coupled with the decrease in the Sd of urea with increasing effluent flow, indicate that equilibration across the filter is unpredictable and may decrease at higher effluent flow rates.
This implies a dynamic but unpredictable loss of filter membrane function with use and this loss may be due to partial filter clotting or membrane fouling due to higher transmembrane pressures [41,42]. Smaller molecules may be more affected by this process because their clearance is diffusion-dependent [40]. The age of the filter probably failed to have an influence in our study because of selection bias (filters that are better functioning and less prone to clotting will last longer). Finally, even in laboratory-based testing of dialyzers, there is marked scatter of these data because of any of the abovementioned factors and changes in transit time due to the effect of internal and back filtration of solutes or water or both [43]. Adsorption of antibiotic to the filter may also contribute to the results observed.

\section{Influence of continuous renal replacement therapy on clearances}

The extracorporeal antibiotic and tazobactam $\mathrm{CL}_{\mathrm{s}}$ observed are consistent with data in the literature [12]; however, we also noted a wide dispersion of results within a single

Table 2 Calculated systemic pharmacokinetic parameters for antibiotics and tazobactam assessed in this study compared with values reported for critically ill patients in the literature

\begin{tabular}{|c|c|c|c|c|c|c|c|c|}
\hline & \multicolumn{2}{|c|}{$\begin{array}{l}\text { Population estimate in this } \\
\text { study (and range in individuals) }\end{array}$} & \multicolumn{2}{|c|}{$\begin{array}{l}\text { Random } \\
\text { effects - BSV (CV\%) }\end{array}$} & \multicolumn{2}{|c|}{ Random error } & \multicolumn{2}{|l|}{$\begin{array}{l}\text { Values reported } \\
\text { in the literature }\end{array}$} \\
\hline & $\mathrm{CL}, \mathrm{mL} /$ minute & Vd & $\mathrm{BSV}_{\mathrm{CL}}$ & $\mathrm{BSV}_{\mathrm{Vd}}$ & RUV (CV\%) & $\mathrm{SD}, \mathrm{mg} / \mathrm{L}$ & $\mathrm{CL}, \mathrm{mL} /$ minute $^{\mathrm{a}}$ & $V d, L^{a}$ \\
\hline \multirow[t]{2}{*}{ Ciprofloxacin } & $58(53-63)$ & $37.7 \mathrm{~L}$ & 10.0 & 34.6 & - & 0.99 & $34-617$ [16-18] & $28-224$ [16-18] \\
\hline & & $(0.32-0.88$ L/kg) & & & & & & \\
\hline \multirow[t]{2}{*}{ Meropenem } & $38(23-95)$ & $17.5 \mathrm{~L}$ & 34.5 & 37.8 & 16.8 & 2.07 & $23-236[10,19-27]$ & $12-212[10,19-27]$ \\
\hline & & $(0.14-0.61 \mathrm{~L} / \mathrm{kg})$ & & & & & & \\
\hline \multirow[t]{2}{*}{ Piperacillin } & $59(37-115)$ & $18.7 \mathrm{~L}$ & 40.9 & 27.2 & 44 & - & $24-438[10,21,28-34]$ & $10-120[10,21,28-34$ \\
\hline & & $(0.14-0.29$ L/kg) & & & & & & \\
\hline \multirow[t]{2}{*}{ Tazobactam } & $113(45-248)$ & $49.3 \mathrm{~L}$ & 52.3 & 34.5 & 41 & - & $22-180[28-30,32,34]$ & $8-60[28-30,32,34]$ \\
\hline & & $(0.54-0.55 \mathrm{~L} / \mathrm{kg})$ & & & & & & \\
\hline \multirow[t]{2}{*}{ Vancomycin } & $25(16-33)$ & $39.7 \mathrm{~L}$ & 27.4 & 24.9 & 16 & 0.032 & 23-73 [35-37] & 20-137 [35-37] \\
\hline & & (0.32-0.74 L/kg) & & & & & & \\
\hline
\end{tabular}




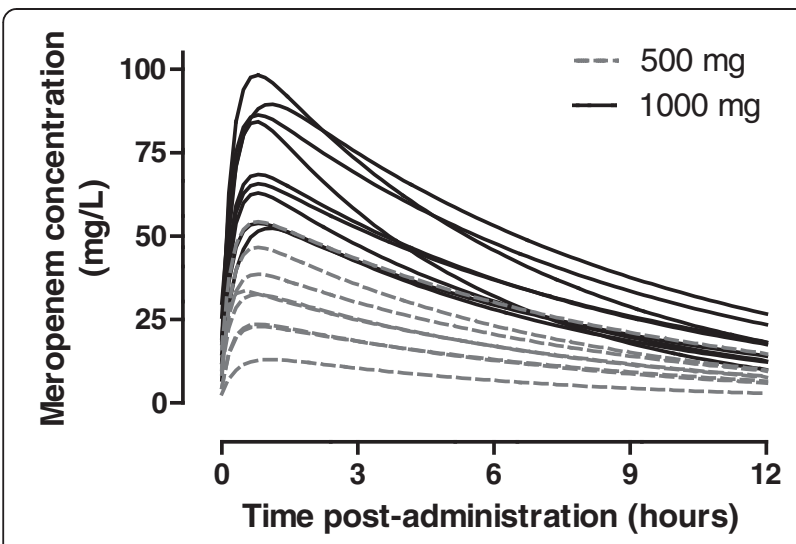

Figure 3 Marked inter-individual and inter-occasion variability in the plasma concentration-time profile of meropenem over 12 hours following 17 doses of either 500 or $1,000 \mathrm{mg}$ to 12 patients. Simulated concentration-time profiles using a single compartment equation are based on the trough concentration, time of infusion, and calculated systemic clearance and volume of distribution for each occasion (performed by using GraphPad Prism version 4.03 for Windows; GraphPad Software, San Diego, CA, USA).

CRRT treatment arm. This had the effect of reducing the clinical and statistical significance of the relatively small differences in concentration that were observed between groups. The reason for this variability requires further investigation and may include differences in renal (and residual function in patients with $\mathrm{AKI}$ ) and non-renal clearance mechanisms.

The CRRT regimen used in this study was that of hemodiafiltration. In both types of CRRT, only the free (unbound) antibiotic can be cleared. The antibiotics assessed in this study are minimally protein-bound [44]: ciprofloxacin $30 \%$ to $40 \%$, meropenem $8 \%$, piperacillin $20-30 \%$, vancomycin $30 \%$ to $40 \%$, and tazobactam $20 \%$ to $30 \%$ (although these values may change in critical illness [26]); so vancomycin CL is predicted to be less than meropenem. Differences in MW can also influence CL in certain CRRT regimens. For example, the partial diffusive $\mathrm{CL}$ used in continuous veno-venous hemodiafiltration will only achieve less vancomycin removal than meropenem; however, we noted inconsistencies in this simple assumption, and possible reasons were discussed above.

\section{Relationship to previous studies}

We observed both inter-individual and inter-occasion (intra-individual) variability in pharmacokinetics. Some variability may depend on the clinical characteristics of patients. For example, in sepsis and critical illness, ciprofloxacin $\mathrm{CL}$ and $\mathrm{Vd}$ are decreased compared with volunteers matched for creatinine CL and weight [18]. Similarly, the Vd and CL of meropenem are lower in critically ill patients with sepsis compared with those with multitrauma, matched for creatinine CL [22]. Finally, the calculated $\mathrm{CL}$ and $\mathrm{Vd}$ for piperacillin is lower after

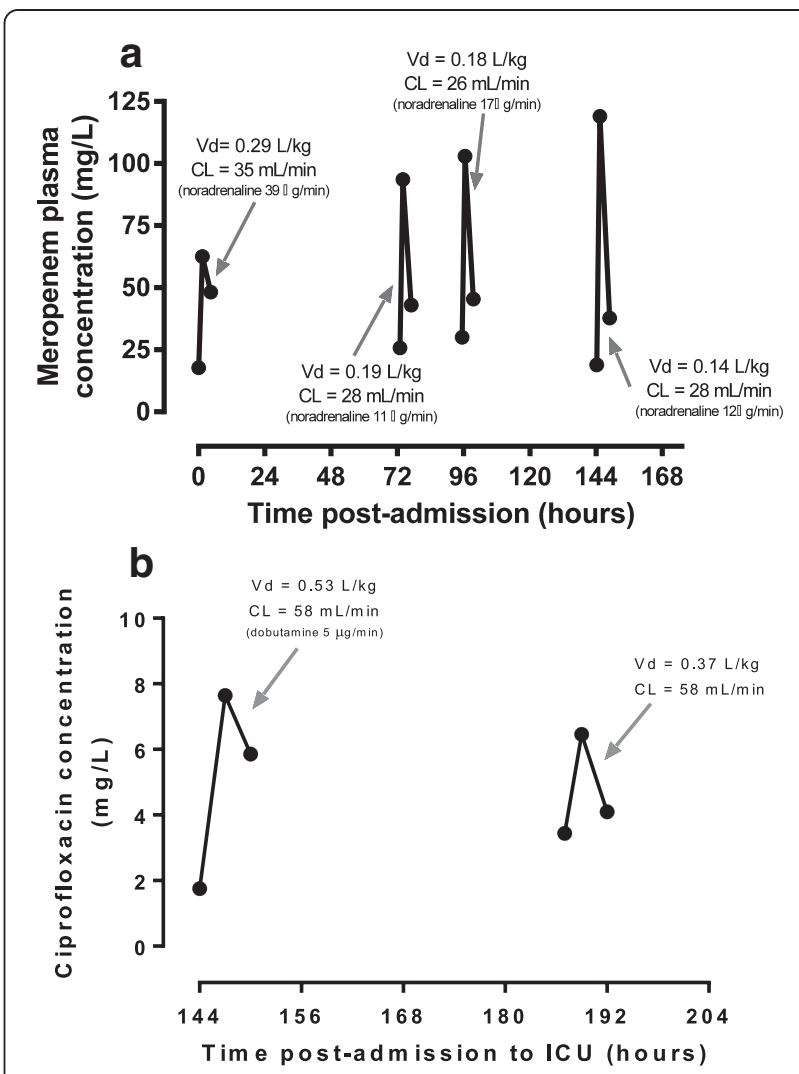

Figure 4 Examples of inter-occasion variability in antibiotic pharmacokinetics. (a) Admission to the intensive care unit (ICU) after coronary artery bypass grafts and valve repair, requiring mechanical ventilation and vasopressors for hypotension (not attributed to sepsis). This patient received higher-intensity continuous renal replacement therapy (CRRT) and did not survive to hospital discharge. Meropenem (1 g) was administered on each occasion. (b) Admission to the ICU from the emergency department and subsequently diagnosis with Klebsiella sepsis associated with a soft tissue infection, complicated by edema and hypoalbuminemia ( $24 \mathrm{~g} / \mathrm{L})$. Treatment included mechanical ventilation, antibiotics, and dopamine infusion (decreasing requirements during admission, not required at the time of second samples). This patient received higher-intensity CRRT and was alive at 90 days post-admission. Ciprofloxacin (200 mg) was administered on both occasions. CL, clearance; Vd, volume of distribution.

multiple doses administered (similar to the context of our data [9]) compared with a single dose [31]. Dynamic changes in residual renal function may specifically contribute to these observations.

\section{Observations with piperacillin and tazobactam}

Piperacillin and tazobactam are co-formulated, and so assessment of their individual pharmacokinetics may inform dosage recommendations. Between-subject variation in pharmacokinetics was observed (Table 2), but with tazobactam there was little variability in the weightnormalized Vd of tazobactam. This suggests that its Vd was influenced primarily by body weight rather than the pathophysiology of critical illness. The $\mathrm{CL}$ and $\mathrm{Vd}$ of 
tazobactam were higher than those of piperacillin. Some studies have shown a similar relationship $[28,30]$ but others have not [29]. Some studies have quantified piperacillin pharmacokinetics and extrapolated these directly to those of tazobactam $[10,21]$, but this appears to be reliable. We noted marked variability in the ratio of piperacillin to tazobactam plasma concentrations as reported by others [28].

\section{Contribution of extracorporeal clearance to the total antibiotic clearance}

Systemic CL and elimination half-life did not differ according to CRRT dose, and so the CRRT prescription may not be useful for guiding antibiotic prescribing. This has been observed previously $[10,22,26]$. There is significant non-renal clearance of vancomycin early in the course of AKI, but this decreases with time [45], which may also contribute to the poor relationship. The contribution of extracorporeal CL to systemic CL was variable. This related to the combined effect of the variability in extracorporeal CL and inter-individual and inter-occasion (despite identical CRRT regimens) variability in systemic pharmacokinetics. In many cases, extracorporeal CL accounted for more than $30 \%$ of the observed systemic $\mathrm{CL}$ for that antibiotic, which is a suggested threshold for adjustment of the dosing regimen. Such variability in CL limits attempts to develop dosing guidelines.

Of note, $C L_{H D F}$ appeared to exceed $C_{s}$ in some cases. The reason for this is not apparent from our data but may relate to an error with either of these calculated values. For example, it has been noted that the delivered effluent flow rate is frequently lower than the prescribed flow rate, and so the calculation of clearance based on the prescribed effluent rate may overstate the actual clearance [41].

\section{Strengths and limitations}

We evaluated the pharmacokinetics of multiple clinically relevant antibiotics administered to patients as part of a prospective multicenter observational study within a large randomized controlled trial. Multiple measurements were obtained, and similarities were observed between our data and the literature. Our data, however, greatly expand our understanding of $\mathrm{CL}, \mathrm{Vd}$, and pharmacokinetics in the setting of CRRT treatment using different CRRT doses and the advantages of randomization and multiple sampling.

Although median extracorporeal CL values were consistent with the literature, the degree of variability was an unexpected finding. All care was taken with the collection of samples by designated staff, subsequent storage, and then analysis using validated methods. However, we cannot exclude that this reflected unmeasured or unknown clinical or mechanical factors occurring during CRRT (for example, an undetected machine error at the time of sampling or a difference between the prescribed and delivered effluent flow rate); however, this also reflects clinical reality. The impact of these factors may have been reduced if clearance was determined from a prolonged collection (over hours) rather than at a point in time, as used in our study.

Our study might be underpowered to detect a true difference in clearance on the basis of CRRT intensity, relating in part, to the selection of patients. The dose ratio of lower- to higher-intensity CRRT effluent is 0.60 per protocol, but in this study the ratio was 0.76 based on the achieved median effluent flow rates. This may narrow the difference between the two groups, although this effect would be lessened by analysis in terms of the actual effluent production rate as performed here rather than according to milliliters per kilogram.

\section{Conclusions}

We have identified marked variability in systemic CL and $\mathrm{Vd}$ of multiple antibiotics in critically ill patients with AKI and also the variable influence of CRRT regimen on extracorporeal CL. Taken together, such variability complicates empiric antibiotic prescribing during CRRT. Ongoing research to further explore what factors contribute to this variability is important. In the meantime, drug monitoring may be the most practical method for ensuring that antibiotic therapeutic targets are achieved in critically ill patients receiving CRRT.

\section{Key messages}

- In this multicenter pharmacokinetic study, the prescribed intensity of continuous renal replacement therapy (CRRT) did not adequately predict solute clearance, volume of distribution, half-life, or the saturation coefficient. This did not appear to be explained by the age of the filter, hematocrit, or serum albumin concentration.

- Using urea and creatinine as biomarkers of membrane function, despite a correlation between effluent flow and clearance from hemodiafiltration $\left(\mathrm{CL}_{\mathrm{HDF}}\right)$, we detected failure of equilibration between dialysate and plasma during higher CRRT intensity.

- The correlation between the saturation coefficient of urea or creatinine with antibiotics was inconsistent, limiting their role as a surrogate measures for clearance.

- This variable pharmacokinetics complicates empiric antibiotic prescribing during CRRT, and ongoing research is required to determine factors that contribute to this variability.

- In the meantime, drug monitoring may be the most practical method for ensuring that antibiotic therapeutic targets are achieved in critically ill patients receiving CRRT. 


\section{Additional file}

Additional file 1: Supplementary data. Inclusion and diagnostic criteria, quantification of beta-lactam antibiotics (laboratory), population pharmacokinetic model development and evaluation, online supplement figures.

\section{Abbreviations}

AKI: acute kidney injury; $C_{E}$ : solute concentration in continuous renal replacement therapy effluent; $C L$ : clearance; $\mathrm{CL}_{\text {HDF }}$ : clearance from hemodiafiltration; $\mathrm{CL}_{s}$ : systemic clearance; $\mathrm{C}_{\mathrm{p}}$ : solute concentration in plasma; CRRT: continuous renal replacement therapy; CV\%: coefficient of variation; IQR: interquartile range; MW: molecular weight; NONMEM: non-linear mixed-effects population modelling software; OFV: (NONMEM) objective function value; Sd: saturation coefficient in the effluent or diafiltrate; $\mathrm{T}_{1 / 2}$ : systemic elimination half-life; $\mathrm{Vd}$ : volume of distribution.

\section{Competing interests}

The authors declare that they have no competing interests.

\section{Authors' contributions}

DMR was involved in the design of this substudy and in the initial analyses of the data and had responsibility for the overall coordination. JAR was involved in the design of this substudy, the laboratory analyses, the initial analyses of the data, the recruitment of patients, recording of clinical details, and compliance with the protocol. $\mathrm{XL}$ and MSR were involved in the design of this substudy, the laboratory analyses, and the initial analyses of the data. $J L$ and RB were involved in the design of this substudy, the design of the RENAL study, the recruitment of patients, recording of clinical details, compliance with the protocol, and the initial analyses of the data. LC and PN were involved in the design of the RENAL study, the recruitment of patients, recording of clinical details, and compliance with the protocol. All authors revised the draft manuscript and approve its submission and contributed to data analysis. All authors read and approved the final manuscript.

\section{Acknowledgments}

The authors thank the research nurses in the participating institutions for recruitment and collection of samples. Financial support was provided by grants from the National Health and Medical Research Council of Australia (352550) and the Health Research Council of New Zealand (06-357). JAR is funded by a Career Development Fellowship (APP1048652) and MSR by a Senior Principal Research Fellowship (APP1002611) from the National Health and Medical Research Council of Australia. The funding organizations had no role in the design of this substudy, analysis, or decision to publish. Written informed consent was obtained from the patient or their responsible surrogate for publication in this article. The consent form is held by the authors and is available for review by the Editor-in-Chief.

\section{Author details}

${ }^{1}$ Burns Trauma and Critical Care Research Centre, The University of Queensland, Level 3 Ned Hanlon Building, Royal Brisbane and Women's Hospital, Butterfield Street, Brisbane, Queensland 4029, Australia. ${ }^{2}$ Therapeutics Research Centre, School of Medicine, University of Queensland, Princess Alexandra Hospital, Ipswich Road, Brisbane, Queensland 4102, Australia. ${ }^{3}$ University of South Australia, City East Campus, GPO Box 2471, Adelaide, South Australia 5000, Australia. ${ }^{4}$ The Queen Elizabeth Hospital, 28 Woodville Road, Woodville South, Adelaide, South Australia 5011, Australia. ${ }^{5}$ Department of Intensive Care Medicine, Level 3 Ned Hanlon Building, Royal Brisbane and Women's Hospital, Butterfield Street, Brisbane, Queensland 4029, Australia. 'Intensive Care Unit, St Vincent's Hospital, Victoria Street, Darlinghurst, NSW 2010, Australia. 'Intensive Care Unit, Nepean Hospital, Derby Street, Kingswood, NSW 2747, Australia. ${ }^{8}$ Department of Intensive Care, Austin Health, 145 Studley Road, Heidelberg, Victoria 3084, Australia.

Received: 13 October 2014 Accepted: 17 February 2015 Published online: 13 March 2015

\section{References}

1. Bagshaw SM, Uchino S, Bellomo R, Morimatsu H, Morgera S, Schetz M, et al. Septic acute kidney injury in critically ill patients: clinical characteristics and outcomes. Clin J Am Soc Nephrol. 2007;2:431-9.

2. Parmar A, Langenberg C, Wan L, May CN, Bellomo R, Bagshaw SM. Epidemiology of septic acute kidney injury. Curr Drug Targets. 2009;10:1169-78.

3. Roberts JA. Using PK/PD to optimize antibiotic dosing for critically ill patients. Curr Pharm Biotechnol. 2011;12:2070-9.

4. Roberts JA, Paul SK, Akova M, Bassetti M, De Waele JJ, Dimopoulos G, et al. DALI: defining antibiotic levels in intensive care unit patients: are current beta-lactam antibiotic doses sufficient for critically ill patients? Clin Infect Dis. 2014,58:1072-83.

5. Roberts JA, Kruger P, Paterson DL, Lipman J. Antibiotic resistance-what's dosing got to do with it? Crit Care Med. 2008;36:2433-40.

6. Roberts DM. The relevance of drug clearance to antibiotic dosing in critically ill patients. Curr Pharm Biotechnol. 2011;12:2002-14.

7. Ulldemolins $\mathrm{M}$, Rello J. The relevance of drug volume of distribution in antibiotic dosing. Curr Pharm Biotechnol. 2011;12:1996-2001.

8. Choi G, Gomersall CD, Tian Q, Joynt GM, Freebairn R, Lipman J. Principles of antibacterial dosing in continuous renal replacement therapy. Crit Care Med. 2009;37:2268-82

9. Roberts DM, Roberts JA, Roberts MS, Liu X, Nair P, Cole L, et al. Variability of antibiotic concentrations in critically ill patients receiving continuous renal replacement therapy - a multicentre pharmacokinetic study. Crit Care Med. 2012;40:1523-8.

10. Seyler L, Cotton F, Taccone FS, De Backer D, Macours P, Vincent $J$, et al. Recommended beta-lactam regimens are inadequate in septic patients treated with continuous renal replacement therapy. Crit Care. 2011;15:R137.

11. Roberts JA, Ulldemolins M, Roberts MS, McWhinney B, Ungerer J, Paterson $\mathrm{DL}$, et al. Therapeutic drug monitoring of beta-lactams in critically ill patients: proof of concept. Int J Antimicrob Agents. 2010;36:332-9.

12. Li AM, Gomersall CD, Choi G, Tian Q, Joynt GM, Lipman J. A systematic review of antibiotic dosing regimens for septic patients receiving continuous renal replacement therapy: do current studies supply sufficient data? J Antimicrob Chemother. 2009;64:929-37.

13. Bellomo R, Cass A, Cole L, Finfer S, Gallagher M, Lo S, et al. Intensity of continuous renal-replacement therapy in critically ill patients. N Engl J Med. 2009:361:1627-38.

14. Schetz $M$, Ferdinande $P$, Van den Berghe $G$, Verwaest $C$, Lauwers $P$. Pharmacokinetics of continuous renal replacement therapy. Intensive Care Med. 1995;21:612-20.

15. Boeckmann AJ, Sheiner LB, Beal SL. NONMEM Users Guide - Part V: Introductory Guide. San Francisco, CA: University of California; 1994.

16. Conil JM, Georges B, de Lussy A, Khachman D, Seguin T, Ruiz S, et al. Ciprofloxacin use in critically ill patients: pharmacokinetic and pharmacodynamic approaches. Int J Antimicrob Agents. 2008;32:505-10.

17. Malone RS, Fish DN, Abraham E, Teitelbaum I. Pharmacokinetics of levofloxacin and ciprofloxacin during continuous renal replacement therapy in critically ill patients. Antimicrob Agents Chemother. 2001;45:2949-54.

18. Forrest A, Ballow CH, Nix DE, Birmingham MC, Schentag JJ. Development of a population pharmacokinetic model and optimal sampling strategies for intravenous ciprofloxacin. Antimicrob Agents Chemother. 1993;37:1065-72.

19. Thalhammer F, Schenk P, Burgmann H, El Menyawi I, Hollenstein UM, Rosenkranz AR, et al. Single-dose pharmacokinetics of meropenem during continuous venovenous hemofiltration. Antimicrob Agents Chemother. 1998;42:2417-20

20. Thalhammer F, Horl WH. Pharmacokinetics of meropenem in patients with renal failure and patients receiving renal replacement therapy. Clin Pharmacokinet. 2000;39:271-9.

21. Taccone FS, Laterre PF, Dugernier T, Spapen H, Delattre I, Wittebole X, et al. Insufficient beta-lactam concentrations in the early phase of severe sepsis and septic shock. Crit Care. 2010;14:R126.

22. Isla A, Rodriguez-Gascon A, Troconiz IF, Bueno L, Solinis MA, Maynar J, et al. Population pharmacokinetics of meropenem in critically ill patients undergoing continuous renal replacement therapy. Clin Pharmacokinet. 2008;47:173-80.

23. Krueger WA, Schroeder TH, Hutchison M, Hoffmann E, Dieterich HJ, Heininger A, et al. Pharmacokinetics of meropenem in critically ill patients with acute renal failure treated by continuous hemodiafiltration. Antimicrob Agents Chemother. 1998;42:2421-4.

24. Giles LJ, Jennings AC, Thomson AH, Creed G, Beale RJ, McLuckie A. Pharmacokinetics of meropenem in intensive care unit patients receiving 
continuous veno-venous hemofiltration or hemodiafiltration. Crit Care Med. 2000;28:632-7.

25. Tegeder I, Neumann F, Bremer F, Brune K, Lotsch J, Geisslinger G. Pharmacokinetics of meropenem in critically ill patients with acute renal failure undergoing continuous venovenous hemofiltration. Clin Pharmacol Ther. 1999;65:50-7.

26. Robatel C, Decosterd LA, Biollaz J, Eckert P, Schaller MD, Buclin T. Pharmacokinetics and dosage adaptation of meropenem during continuous venovenous hemodiafiltration in critically ill patients. J Clin Pharmacol. 2003:43:1329-40

27. Ververs TF, van Dijk A, Vinks SA, Blankestijn PJ, Savelkoul JF, Meulenbelt J, et al. Pharmacokinetics and dosing regimen of meropenem in critically il patients receiving continuous venovenous hemofiltration. Crit Care Med. 2000:28:3412-6.

28. van der Werf TS, Mulder PO, Zijlstra JG, Uges DR, Stegeman CA. Pharmacokinetics of piperacillin and tazobactam in critically ill patients with renal failure, treated with continuous veno-venous hemofiltration (CWH). Intensive Care Med. 1997;23:873-7.

29. Valtonen M, Tiula E, Takkunen O, Backman JT, Neuvonen PJ. Elimination of the piperacillin/tazobactam combination during continuous venovenous haemofiltration and haemodiafiltration in patients with acute renal failure. J Antimicrob Chemother. 2001:48:881-5.

30. Buck C, Bertram N, Ackermann T, Sauerbruch T, Derendorf H, Paar WD. Pharmacokinetics of piperacillin-tazobactam: intermittent dosing versus continuous infusion. Int J Antimicrob Agents. 2005;25:62-7.

31. Capellier G, Cornette C, Boillot A, Guinchard C, Jacques T, Blasco G, et al. Removal of piperacillin in critically ill patients undergoing continuous venovenous hemofiltration. Crit Care Med. 1998;26:88-91.

32. Arzuaga A, Maynar J, Gascon AR, Isla A, Corral E, Fonseca F, et al. Influence of renal function on the pharmacokinetics of piperacillin/tazobactam in intensive care unit patients during continuous venovenous hemofiltration. J Clin Pharmacol. 2005;45:168-76.

33. Krueger WA, Neeser G, Schuster H, Schroeder TH, Hoffmann E, Heininger A, et al. Correlation of meropenem plasma levels with pharmacodynamic requirements in critically ill patients receiving continuous veno-venous hemofiltration. Chemotherapy. 2003:49:280-6.

34. Mueller SC, Majcher-Peszynska J, Hickstein H, Francke A, Pertschy A, Schulz $M$, et al. Pharmacokinetics of piperacillin-tazobactam in anuric intensive care patients during continuous venovenous hemodialysis. Antimicrob Agents Chemother. 2002:46:1557-60.

35. DelDot ME, Lipman J, Tett SE. Vancomycin pharmacokinetics in critically ill patients receiving continuous venovenous haemodiafiltration. Br J Clin Pharmacol. 2004;58:259-68

36. Boereboom FT, Ververs FF, Blankestijn PJ, Savelkoul TJ, van Dijk A. Vancomycin clearance during continuous venovenous haemofiltration in critically ill patients. Intensive Care Med. 1999;25:1100-4.

37. Santre C, Leroy O, Simon M, Georges H, Guery B, Beuscart C, et al. Pharmacokinetics of vancomycin during continuous hemodiafiltration. Intensive Care Med. 1993;19:347-50

38. Bouchard J, Roberts DM, Roy L, Ouellet G, Decker BS, Mueller BA, et al. Principles and operational parameters to optimize poison removal with extracorporeal treatments. Semin Dial. 2014;27:371-80.

39. Padrini R, Canova C, Conz P, Mancini E, Rizzioli E, Santoro A. Convective and adsorptive removal of beta2-microglobulin during predilutional and postdilutional hemofiltration. Kidney Int. 2005;68:2331-7.

40. Brunet S, Leblanc M, Geadah D, Parent D, Courteau S, Cardinal J. Diffusive and convective solute clearances during continuous renal replacement therapy at various dialysate and ultrafiltration flow rates. Am J Kidney Dis. 1999;34:486-92.

41. Claure-Del Granado R, Macedo E, Chertow GM, Soroko S, Himmelfarb J, Ikizler TA, et al. Effluent volume in continuous renal replacement therapy overestimates the delivered dose of dialysis. Clin J Am Soc Nephrol. 2011;6:467-75.

42. Sakiyama R, Ishimori I, Akiba T, Mineshima M. Effect of blood flow rate on internal filtration in a high-flux dialyzer with polysulfone membrane. J Artif Organs. 2012;15:266-71.

43. Schneditz D, Zierler E, Jantscher A, Vanholder R, Eloot S. Internal filtration in a high-flux dialyzer quantified by mean transit time of an albumin-bound indicator. ASAIO J. 2013;59:505-11.
44. Pea F, Viale P, Pavan F, Furlanut M. Pharmacokinetic considerations for antimicrobial therapy in patients receiving renal replacement therapy. Clin Pharmacokinet. 2007;46:997-1038.

45. Macias WL, Mueller BA, Scarim SK. Vancomycin pharmacokinetics in acute renal failure: preservation of nonrenal clearance. Clin Pharmacol Ther. 1991;50:688-94.

\section{Submit your next manuscript to BioMed Central and take full advantage of:}

- Convenient online submission

- Thorough peer review

- No space constraints or color figure charges

- Immediate publication on acceptance

- Inclusion in PubMed, CAS, Scopus and Google Scholar

- Research which is freely available for redistribution 